\title{
Cast Tube Assay: A 3-D in vitro assay for visualization and quantification of horizontal chemotaxis and cellular invasion
}

\author{
Breffeni Constantine Whitehead, Deon Bezuidenhout, Cindy Chokoza, Neil Hamer Davies, and \\ Kyle Peter Goetsch \\ Cardiovascular Research Unit, Chris Barnard Division of Cardiothoracic Surgery, University of Cape Town, \\ Department of Health Sciences, Cape Town, South Africa
}

BioTechniques 61:66-72 (August 2016) doi 10.2144/000114442

Keywords: chemotaxis, cellular invasion, hydrogel, three-dimensional (3-D) in vitro assay

Supplementary material for this article is available at www.BioTechniques.com/article/114442.

Directed cell motility, as controlled by soluble factors, is crucial for many biological processes, including development, cancer progression, and wound healing. The use of directed cell motility also shows promise for applications in regenerative medicine such as therapeutic angiogenesis. Unfortunately, current in vitro 3-D migration and invasion models limit our understanding and application of these processes. Here, we present a novel and cost-effective 3-D chemotaxis assay for assessing the invasive response of cells to a chemoattractant extracellular matrix (ECM). Our system takes advantage of a custom-casting chamber to set two gels in contact with each other along a defined front, one containing a suitable chemoattractant and the other the cells. Rotation of the chamber allows easy visualization of invasion across the interface. The effectiveness of the assay was demonstrated by studying the invasion of both human dermal fibroblasts (FBs) and smooth muscle cells (SMCs) into a polyethylene glycol (PEG) hydrogel containing basic fibroblast growth factor (bFGF). Incorporation of bFGF resulted in significantly increased and directional invasion for both cell groups.

Cellular invasion plays a key role in many important biological processes such as development (1), immune function, wound healing (2), and cancer progression (3). In development, migration is responsible for formation of various body systems $(1,4)$. In the immune system, effector cells must travel to and invade sites of infection or injury in response to chemical distress signals. Closely linked to this is the process of wound healing, where invasion of fibroblasts (FBs) into the wound site following the inflammatory response is important for scar formation and wound closure (2).

Growth factor-mediated invasion also serves as the basis for angio- genesis, a process with many potential therapeutic applications $(5,6)$, especially in the context of regenerative medicine, where perfusion of implanted tissues and structures is a significant challenge.

As these processes are complex, there is much to be gained through a better understanding of cell migration, and its interaction with signaling molecules. To achieve this, assay systems capable of modeling these events under controlled conditions are required. An ideal solution is to work in vivo; however, the ethical challenges of animal work limit the throughput of such experiments. Furthermore, gaining real-time data on cellular movement in in vivo models is extremely demanding. These challenges highlight the need for in vitro models that mimic in vivo conditions as closely as possible.

2-D cell culture is well-established, and there exist several 2-D chemotaxis assays (7-11). While these assays are useful in their own right, physiological migration and invasion typically occur in 3-D, a largely different environment. Several studies have shown that 2-D and 3-D cultured cells differ in morphology, and gene expression (12). Additionally, ligand distribution in 3-D systems can differ from 2-D systems (13). 3-D migration also involves degra-

\section{METHOD SUMMARY}

Our Cast Tube assay uses an easily produced independent chamber to cast two gels in contact with each other along a defined plane; one containing a cellular sample and the other an appropriate chemoattractant. Gels are stacked one on top of another to generate even interfaces, after which the chamber is rotated to overcome the challenges of tracking vertical cell migration. 
dation of the extracellular matrix (ECM) surrounding the motile cells (14), and parameters such as the stiffness of the surrounding matrix have a profound effect on a cell's ability to migrate (15). 2-D culture also creates an artificial polarity (16) where adhesion moleculebased signaling is present on only one surface of the cell. These differences limit our ability to accurately model physiological migration using 2-D systems.

ECM mimics and gel systems provide a means of studying cell movement in 3-D in vitro, and several such assays have been developed. One of the most widely used is the Boyden chamber, or Transwell (Corning) system, which quantifies chemotaxis by counting cells that migrate through an ECM-coated membrane. While this approach more closely represents a 2-D model of invasion, the ECM coating can be thickened to a full layer to better simulate a 3-D environment (16-18). While the assay is robust, provides useful information about the interaction of a cell type with a specific factor, and is suitable for high-throughput screening, it does have limitations. The ability of cells to pass the final membrane and subsequently attach to its underside is independent of migration through the gel above, leading to possible inaccuracies when quantifying chemotaxis (19). In addition, the assay does not allow visualization of the morphology of cells as they move or identification of any migratory patterns (20).

Other cell migration assays exist, some of which utilize custom-built chambers or microfluidic systems. While many of these are commercially available, such as ibidi's 3-D $\mu$-slide chambers, others require substantial polymer-shaping facilities to produce chambers (21). These chambers can provide visualization of cell migration in real time using standard microscopy techniques, as well as a means of quantifying migration by cell tracking as opposed to counting, and also facilitate more complex simulations such as the application of shear stress in assays. Unfortunately, the expense and/or complexity of these systems limit their availability. Specialized slides and chambers can make experiments using large numbers of samples

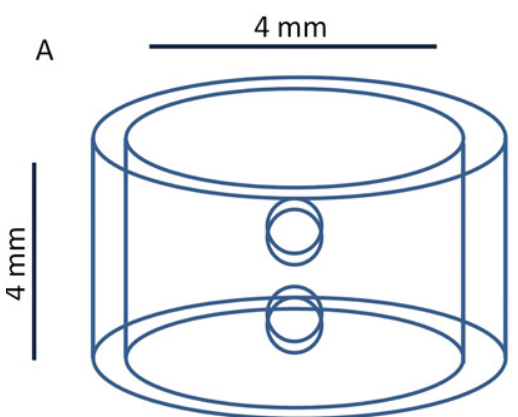

B

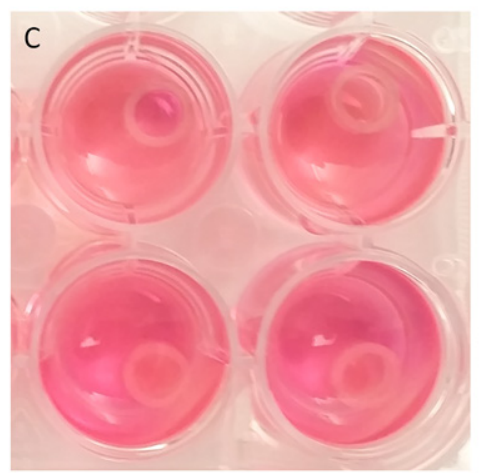

D
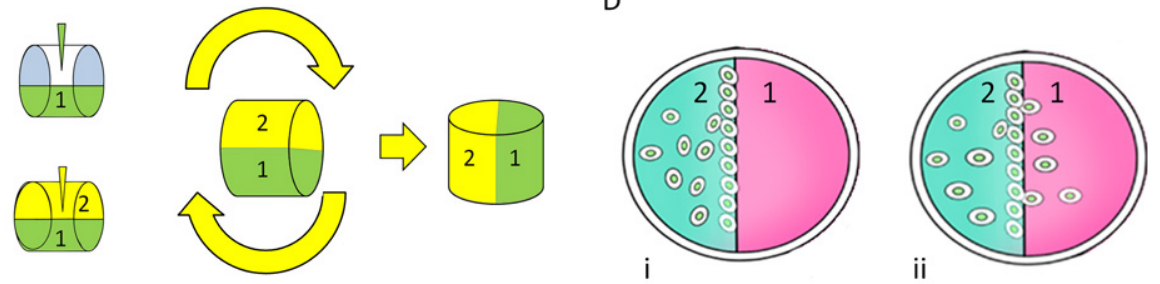

Figure 1. Cast Tube Assay design. (A) Schematic of the cast tube chamber with $4 \mathrm{~mm}$ internal diameter by $4 \mathrm{~mm}$ height. (B) Diagram showing gel casting followed by rotation of the chamber for incubation and visualization; "1" indicates the gel containing chemoattractant, and " 2 " indicates the gel containing cells. (C) Image of cast tubes in a tissue culture plate. (D) Schematic cross-section of the Cast Tube Assay showing cells at seeding ( $i$ ) and after migration (ii); " 1 " indicates chemoattractant gel, and " 2 " indicates the gel containing cells.

cumbersome, though this problem can be alleviated to some degree by running multiple assays on a single chip or slide. Another limitation with closed micro-chambers is the difficulty associated with post-experimental staining and labeling. Therefore, an assay that allows for easy and effective visualization without being complex or expensive to produce and use is attractive. Some models meeting these criteria do exist, such as the agarose spot assay (22); however, they are limited in that migrating cells are still adherent to a 2-D substrate and then migrate into a 3-D gel; this is not representative of true 3-D migration.

Here we present a novel 3-D chemotaxis assay, termed the Cast Tube Assay, that allows easy visualization and quantification of cell invasion. It is also economical and allows the study of several analytes simultaneously. Finally, the Cast Tube Assay allows modeling of invasion from one gel system into another and is amenable to post-experimental staining. We used a polyethylene glycol (PEG) hydrogel system with both human smooth muscle cells (SMCs) and human dermal FBs attracted to basic fibroblast growth factor (bFGF) to prove the system conceptually by quantifying invasion for up to 5 days.

\section{Materials and methods}

\section{PEG hydrogel formation}

Vinyl sulfone derivatized 4-arm PEG with a molecular weight of $20 \mathrm{kDa}$ (20PEG$4 \mathrm{VS}$ ) was prepared as described previously (23). Gels were formed by a Michael-type conjugate addition between equimolar ratios of vinyl sulfone groups of the derivatized PEG and thiols of cysteine residues on a bis-cysteine matrix metalloproteinase (MMP)-sensitive peptide. A peptide sequence (GCREGPQGIWGQERCG; 1733 Da) (GenScript USA Inc., Piscataway, NJ) degradable by a range of MMP's (24) was used to form 3.5\% (mass/mass) gels. In order to bind growth factors, $1.5 \%(\mathrm{~m} / \mathrm{m})$ of the PEG was replaced with heparin acrylate (HepAc) (manuscript in preparation), which is also able to bind to the crosslinker peptide and form part of the polymeric hydrogel. Prior to addition of HepAc and crosslinker, PEG was incubated with RGDS adhesion substrate (GCGYGRGDSPG; $1025 \mathrm{Da}$ ) in a molar ratio of $12.5: 120 \mathrm{PEG}-4 \mathrm{VS}$ for $30 \mathrm{~min}$ at $37^{\circ} \mathrm{C}$. Gels were brought up to volume using iso-osmotic phosphate buffered 
saline (IPBS, $\mathrm{pH}$ 7.5) before addition of crosslinker peptide. When growth factor was added to the gel, it was added with the IPBS before the addition of the crosslinking peptide.

\section{Cell culture}

Human dermal FBs and human SMCs used in this study were isolated as previously described (24). Dulbecco's Modified Eagle Medium (DMEM) (D5523; Sigma-Aldrich, St. Louis, MO) and MCDB medium (M8537; Sigma-Aldrich), both $\mathrm{pH}$ 7.4 and supplemented with $10 \%$ (v/v) fetal calf serum (FCS) (26140-079; Thermo Scientific/Life Technologies, Waltham, $\mathrm{MA})$, and $2 \%(\mathrm{v} / \mathrm{v})$ penicillin/streptomycin (Pen/strep) (15140122; Life Technologies) were used for cell culture. Cells were kept below passage 10. Growth medium was replaced every 3 days for standard culture and once every 4 days for cast tubes.

\section{2-D chemotaxis}

We utilized Ibidi's 2-D $\mu$-Slide chemotaxis assay system (80306; Ibidi, Planegg, Germany) according to the manufacturer's specifications using FBs cultured in DMEM. Human recombinant bFGF (100-18b; Peprotech, Rocky Hill, CT) was used at a final concentration of either 10 or $20 \mathrm{ng} / \mathrm{mL}$. The recommended cell seeding density of $3 \times 10^{6}$ was observed. Migration was monitored over 24 h using standard inverted stage microscopy

A

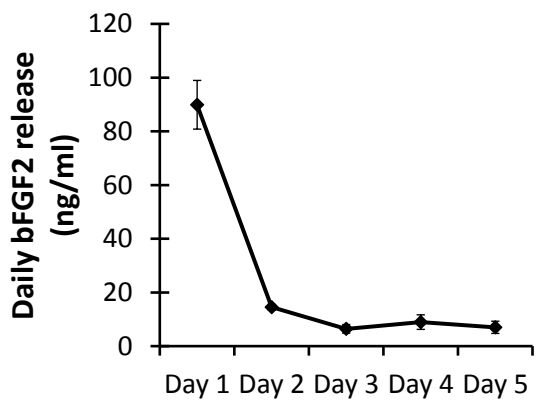

\begin{tabular}{|l|l|}
\hline Average daily release post day 1: & $9.23 \mathrm{ng}$ \\
\hline
\end{tabular}

Figure 2. Gel characterization and 2-D chemotaxis. (A) Basic fibroblast growth factor (bFGF) release from heparinized polyethylene glycol (PEG) hydrogels with average daily release after day 1 (3.5\% PEG, 1.5\% heparin acrylate (HepAc), $50 \mu \mathrm{g} / \mathrm{mL}$ gel samples). Plates were read at $450 \mathrm{~nm}(n=4)$. (B) Average cellular velocity of fibroblasts (FBs) at 10 and $20 \mathrm{ng} / \mathrm{mL}$ bFGF tracked over 24 h. (C) Forward migration index (FMI) for cells in the presence of differing growth factor concentrations. Error bars represent standard error of the mean (SEM); * indicates significant difference from control; \# indicates significant difference between experimental groups; $(P<0.05)$. with an incubated stage at $100 \times$ magnification with 1 image recorded every 20 min via a Motic Moticam T microscope tablet (Motic, Hong Kong, China) camera connected to a Leica DMIRBE microscope (Leica, Wynberg, South Africa). A minimum of 26 cells were analyzed per experiment by manually tracking the cell paths using the chemotaxis and migration toolkit plug-in for ImageJ image processing software (www.ibidi. de/applications/ap_chemo.html).

\section{ELISA}

Fifty microliters PEG hydrogel was formed with $2.5 \mu \mathrm{g} \mathrm{bFGF} \mathrm{for} \mathrm{a} \mathrm{final} \mathrm{gel}$ concentration of $50 \mathrm{ng} / \mu \mathrm{l}$. This was then aliquoted into $10 \mu \mathrm{L}$ droplets and allowed to set. Gels were placed in $500 \mu \mathrm{L} 1 \%$ BSA (B2518; Sigma) PBS, pH 7.4 and incubated on a shaker at $37^{\circ} \mathrm{C}$. Every $24 \mathrm{~h}$, the gel in each sample was transferred to a fresh tube containing 500 $\mu \mathrm{L}$ BSA PBS, and the old sample was flash-frozen and stored at $-20^{\circ} \mathrm{C}$ until the ELISA was performed. Samples were taken over 5 days $(n=4)$. A commercial ELISA system (DY233-05, DuoSet; R\&D Systems, Minneapolis, MN) was used according the manufacturer's specifications. Samples were diluted 1:50 to ensure values were within the established standard curve $(0-2 \mathrm{ng} / \mathrm{mL}$ generated using a serial dilution of stock solution provided by the manufacturer) Plates

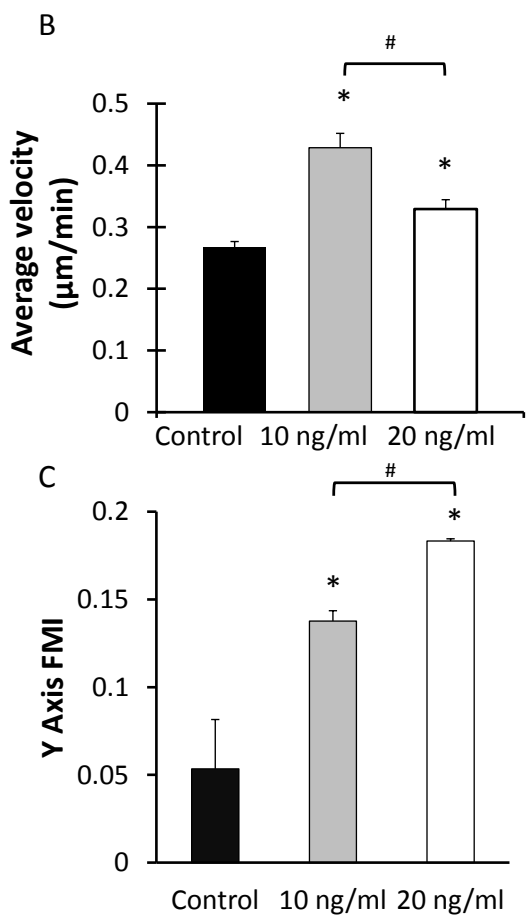

were read on a Bio-Rad microplate reader (170-6800; BioRad, Rosebank, Johannesburg, South Africa) using a 595 nm filter (170-6914; BioRad).

\section{Rheology}

One-hundred-twenty microliter gels (3.5\% PEG, 1.5\% HepAc) were cast between spaced, Sigmacote coated glass plates (Sigma-Aldrich) and allowed to set for $40 \mathrm{~min}$ at room temperature. The gel disks ( $2 \mathrm{~cm}$ diameter by $1 \mathrm{~mm}$ thick) were placed in water to swell overnight, then removed from the plates and placed in water in $3.5 \mathrm{~cm}$ Petri dishes for 3 hours to ensure complete swelling of hydrogels. Gel stiffness was subsequently measured using a shear stress rheometer (TA INSTRUMENTS AR 2000 EX Rheometer; New Castle, DE) with a steel flat plate measuring system in oscillating mode. A constant strain of $0.05 \%$ and a gap of $400 \mu \mathrm{m}$ were used. Readings were taken over a frequency range of $0.5-5 \mathrm{~Hz}$, with $1 \mathrm{~Hz}$ used for plotting the graph (23).

\section{Live/dead assay}

Cell viability was assessed using ethidium homodimer 1 (EthH1) (Thermo Scientific/ Life Technologies) and the calcein-AM LIVE/DEAD kit (L-3224; Thermo Scientific/Life Technologies) to stain dead and living cells, respectively. Stains were applied to cast tubes at $2 \mu \mathrm{M}$ and $4 \mu \mathrm{M}$ for EthH1 and calcein, respectively, for 1 hour on a slow shaker. Viability was assessed by counting live versus dead cells using a standard fluorescence microscope (Zeiss Axiovert 200M) with an AxioCam HRm (high resolution monochrome camera for image capture) (Zeiss, Milnerton, Cape Town, South Africa). Images were taken at 100x using Alex 488 (green) and Cy3 (red) fluorescent filters; 3 fields were assessed per tube.

\section{Cast Tube Assay (3-D chemotaxis)}

Cast tubes were prepared from medical grade silicon tubing $(4 \mathrm{~mm} \times 4 \mathrm{~mm}$ ) (SI-4x7-1-25; Parker, Cleveland, $\mathrm{OH}$ ) and half filled $(45 \mu \mathrm{L})$ with PEG gel $(3.5 \%$ PEG, 1.5\% HepAc) containing $50 \mathrm{ng} / \mu \mathrm{l}$ bFGF. Gels were set for 1 hour at $37^{\circ} \mathrm{C}$. Gels containing cells (20,000/tube) were then cast above the chemoattractant gels. After allowing the second gel to set, the tubes were incubated in MCDB 
medium (10\% FCS, 2\% Pen/strep) at $37^{\circ} \mathrm{C}$. Medium was replaced every 4 days. Control tubes with an initial gel containing no growth factor were used for comparison. Tubes were imaged at Days 3 and 5 using an inverted stage microscope at 100x and 200x magnification. Live/dead staining was performed at Day 7. A $2.5 \mathrm{~mL}$ aliquot of medium was added per Cast Tube Assay. Migration in cast tubes was analyzed by measuring the invaded distance from a defined gel front using the ImageJ line measuring tool (Supplementary Protocol). This was done on randomly selected fields of view with all distinct cells in the plane of the image being recorded. A minimum of seven sprouts were selected per image.

\section{Immunocytochemistry}

Cast Tube Assays were fixed with 4\% paraformaldehyde overnight. The cells were stained with Alexa Fluor 568 phalloidin (Invitrogen Molecular probes, Eugene, OR) for $4 \mathrm{~h}$ at room temperature to allow complete penetration into the hydrogels. The cells were also stained with the nuclear stain Hoechst 33342 dye (Sigma-Aldrich, Chemie $\mathrm{GmbH}$, Steinheim, Germany) for 30 min at room temperature. Images were acquired with a Zeiss 510 LSM Meta confocal microscope. Z-stacks were created with the Zen 2012 software (Zeiss).

\section{Statistical analysis}

Two-tailed Student's t-tests were used to compare means in both 2-D and 3-D migration assays. A Rayleigh test for vector data in 2-D analysis was used to determine the significance of the migrational directionality. $P<0.05$ was considered to be significant. Error bars represent standard error of the mean (SEM).

\section{Results and discussion}

Our primary goals for developing this assay were: (i) simplified visualization and quantification of cell migration, (ii) modeling of true 3-D cell migration, and (iii) examination of invasion from one gel into another. A practical solution was to cast two gels, one atop the other, where one contains cells and the other contains an appropriate chemoattractant. The interface between the two gel systems therefore provides a means of measuring cellular invasion by simply observing the distance cells have traveled into the second gel. The problem with such a system is that displacement of cells, and indeed determination of the gel interface, is difficult in the vertical plane, requiring Z-stacking and computation-dependent depth analysis.

To resolve this issue, our assay takes advantage of an external casting chamber consisting of a simple silicon tube with a pair of vent holes in one side (Figure 1A) and the other openings blocked using sterile laboratory parafilm. The tube is placed on its side, and gels are cast sequentially inside the tube via the vent holes. Once both gels have set, the blocks are removed, and the tube is rotated (Figure 1B), allowing easy visualization of horizontal migration through the bottom of the system (Figure 1D). Tubes are placed in a standard 24-well tissue culture plate with appropriate culture medium for incubation. This allows analysis of multiple samples on

\section{A}
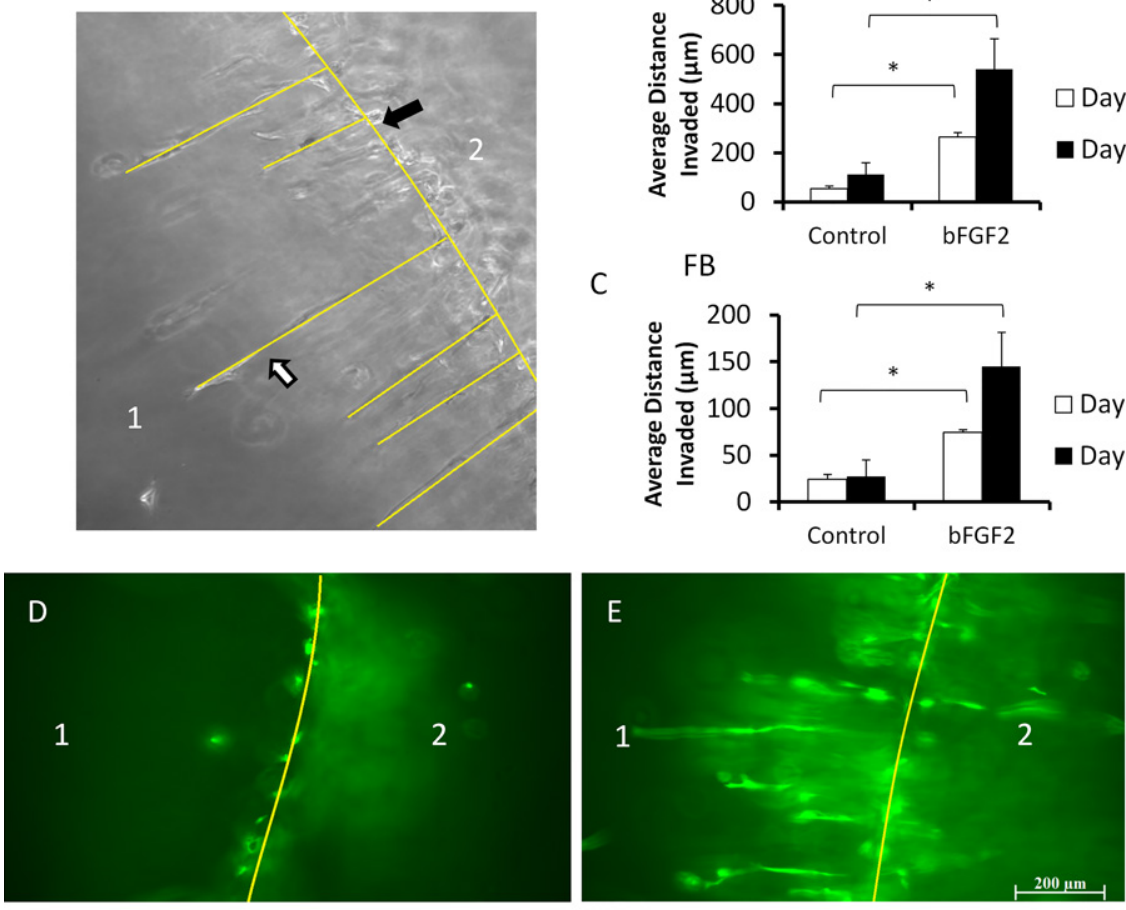

Figure 3. 3-D chemotaxis in Cast Tube Assay. (A) Image showing quantification of smooth muscle cell (SMC) cast tube containing $50 \mu \mathrm{g} / \mathrm{mL}$ basic fibroblast growth factor (bFGF) at Day 5 (standard phase contrast microscope at 100x). (Note that this is a single image representing data from four independent experiments). Black arrow indicates defined gel interface; white arrow indicates measurement of distance invaded. (B) Quantification of SMC invasion into cast tubes at Days 3 and 5 via assessment of average distance invaded. (C) Quantification of fibroblast (FB) invasion into cast tubes at Days 3 and 5 via assessment of average distance invaded. (D) SMC control tube after calcein staining. (E) SMC cast assay tube containing bFGF after calcein staining [Images in (D) and (E) are 100x; green indicates live cells at Day 7]. In (A), (D), and (E), "1" indicates chemoattractant gel containing bFGF, while "2" indicates the gel containing cells. Error bars represent standard error of the mean (SEM); * indicates significant difference $(P<0.05) ; n=4$ for both cell groups with at least 7 cells observed per repeat. 
A

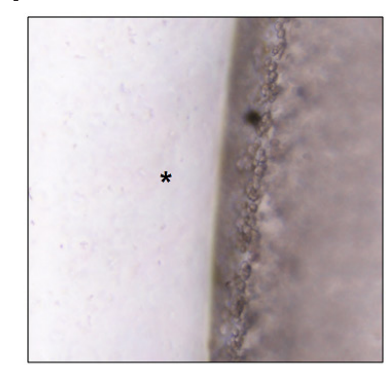

B

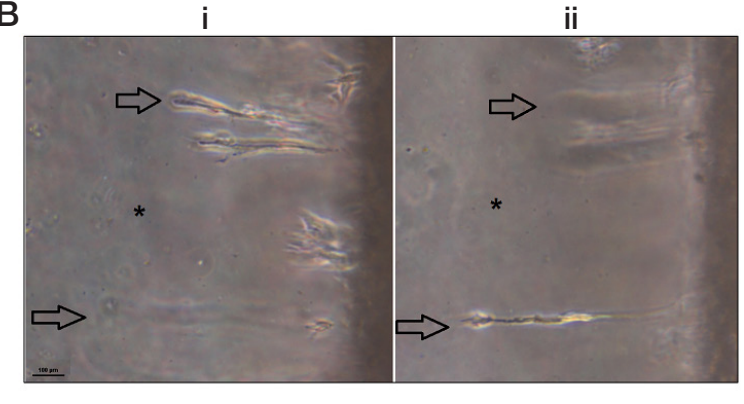

C

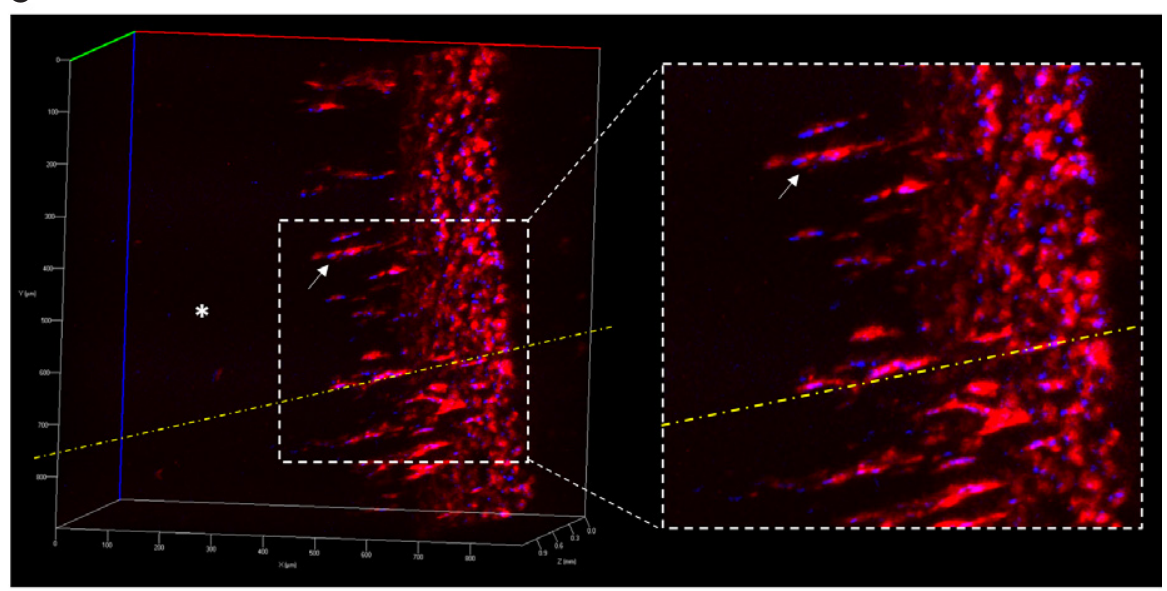

Figure 4. Visualization of 3-D Cast Tube Assay. (A) Initial alignment of cells along the gel interface. (B) Different horizontal planes within the tube assay. (B, i) One brightfield focal plane at $4 \times$ magnification. (B, ii) Different focal plane below that of the first. Arrows represent the same vertical location. Image shows that the sprouts have a linear horizontal migration profile. (C) Confocal microscopy Z-stack showing the invasion of cells in the vertical plane. Yellow dashed line represents one plane as shown in ( $\mathrm{B}, \mathrm{i})$ or $(\mathrm{B}, \mathrm{ii})$. Arrow shows linear sprouts into the hydrogel containing the chemoattractant. * represents hydrogel loaded with basic fibroblast growth factor (bFGF).

tigated its mechanical properties, as well as its ability to bind and release growth factor at a constant rate.

Mechanical characterization of the gel demonstrated an average shear modulus of $200 \pm 39.1 \mathrm{~Pa}$. This value is below the threshold of $300 \mathrm{~Pa}$ at which gel stiffness begins to significantly reduce invasion in a similar MMP-degradable PEG system (15). While this study makes use of a specific gel, any hydrogel system that supports cell migration and some degree of controlled growth factor release could be used, allowing testing of a variety of different matrices that meet these minimal requirements.

Growth factor release from gels containing $50 \mu \mathrm{g} / \mathrm{mL}$ bFGF was assessed by ELISA, showing a consistent daily release of $9.27 \pm 3.68 \mathrm{ng}$ after the large initial release (Figure 2B). A bFGF dose of $10 \mathrm{ng} / \mathrm{mL}$ corresponded to maximal migration of FBs in 2-D (Figure 2C); therefore, this concentration was selected for further use. The motility decrease seen in cells exposed to $20 \mathrm{ng} / \mathrm{mL}$ bFGF
(Figure 2C) is possibly due to the dosage being too high. Many growth factors have a parabolic response curve (27); thus, a very large dose can still induce directional movement, as evidenced by the forward migration index values (FMI) seen in Figure 2-D, but result in less movement as the cells quickly encounter highly concentrated growth factor, leading them to halt their migration.

With our gel system and its capacity to deliver growth factor established, we next sought to test the system by observing cellular invasion into a bFGF containing gel. This invasion was quantified by measuring the average distance cells had invaded into the bFGF hydrogel (Figure 3A) from the interface between the two gels. For both cell lines, the presence of growth factor induced substantially more invasion at both Days 3 and 5 after cells were added to the tubes. On average, SMCs in the presence of growth factor invaded $264 \pm 98 \mu \mathrm{m}$ at Day 3 and 740 $\pm 116 \mu \mathrm{m}$ at Day 5; this was significantly more than the growth factor-negative tubes, in which cells migrated $53 \pm 28$ $\mu \mathrm{m}$ and $112 \pm 39 \mu \mathrm{m}$ at Days 3 and 5 , respectively (Figure $3 \mathrm{~B})(P<0.05$ for both days). The effect was less pronounced for FBs with an average invasion at Day 3 of $75 \pm 36 \mu \mathrm{m}$ and $145 \pm 73 \mu \mathrm{m}$ at Day 5 in the presence of bFGF. This, however, was still significantly more than the control tubes, in which cells migrated only $24 \pm 11 \mu \mathrm{m}$ at Day 3 and $28 \pm 6 \mu \mathrm{m}$ at Day 5 ( $P<0.05$ for both days) (Figure $3 C)$. It should be noted that directional, migratory cell morphology was clearly visible in the growth factor-containing samples, with cells showing an elongated spindle shape, parallel to the direction of the factor gradient (Figure 3A, white arrow).

Cell viability, assessed using live/dead staining, showed no significant increase in cell death in either the growth factor positive or growth factor negative groups (Figure 3, D and E). This was consistent across both cell lines (data not shown) and confirms a lack of cytoxic elements in the assay. It also demonstrates the ease of use of conventional cell staining methods for analysis of cast tube assays.

The gel interface, as well as the cells along the interface, can be easily visualized with brightfield microscopy at $4 \times$ magnification (Figure 4A). Sprouts can be seen invading into the horizontal plane perpendicular to the cell front in a relatively linear fashion toward the chemoattractant-containing hydrogel (Figure 4B). The 3-D nature of the assay makes it difficult to incorporate multiple sprouts into a single view. Thus, to further confirm the manner in which cells invade, we performed a multi-plane Z-stack with confocal microscopy to aid sprout visualization. Cells were labeled with both cytoskeletal and nuclear fluorescent markers (Figure 4C). The multi-plane imaging (Z-stack) allows for quantification of a large area of the gel interface in the vertical plane, with the sprout direction clearly visible. The presence of nuclei in the cellular sprouts confirms that multiple cellular invasion sprouts are being observed and not extensions of long cellular processes; it is also confirms that sprouts are migrating in a relatively perpendicular manner to the original cell front into the opposing hydrogel.

The Cast Tube Assay has several advantages: (i) visualization is extremely quick and easy, and (ii) visualization does 
not require highly specialized equipment or software other than a standard cell laboratory inverted microscope and camera. Data can be gathered as single images, as Z-stacks, or potentially by time-lapse microscopy. Data analysis is similarly efficient as the easily defined starting point (gel interface) allows rapid quantification with no need to track cellspecific cell paths. The assay is amenable to post-experimental staining and parallel experimentation with multiple conditions in a single culture plate. Furthermore, it uses inexpensive materials and requires no more complex facilities than those used for standard tissue culture. Experimental conditions can also be altered in real time by changing the composition of the culture medium used. Placing the culture plate containing the cast tubes on an incubated microscope stage allows real-time observation of invasion. Another benefit of this method is the ability to vary the stiffness of each gel independently, which could be used to model migration of cells between tissue types, for example migration from or into bone tissue.

Here we present a novel 3-D chemotaxis assay system that is relatively inexpensive, simple to use, and allows for rapid visualization and quantification of cell migration. This assay also supports the modification of experimental conditions in real time by changing the properties of the incubation medium, as well as allowing the use of conventional staining techniques. We anticipate that this system will allow more laboratories to conduct 3-D cell culture experiments.

\section{Author contributions}

B.C.W. contributed to experiment and assay design, performed experiments, and wrote the first draft of the manuscript. D.B. developed the PEG hydrogel and edited the manuscript. C.C. contributed to the experimental design and edited the manuscript. N.H.D. supervised the study, contributed to the experimental design, and edited the manuscript. K.P.G. supervised the study, contributed to the experimental design, performed experiments shown in Figure 4, and edited the manuscript.

\section{Acknowledgments}

We would like to thank the staff of the Confocal and Light Microscope Imaging
Facility, University of Cape Town, South Africa for their assistance. This work was supported by grants from the South African Medical Research Council (415626) and the South African National Research Foundation (78802).

\section{Competing interests}

The authors declare no competing interests.

\section{References}

1. Lumsden, A., N. Sprawson, and A. Graham. 1991. Segmental origin and migration of neural crest cells in the hindbrain region of the chick embryo. Development 113:1281-1291.

2. Martin, P. 1997. Wound healing--aiming for perfect skin regeneration. Science 276:75-81.

3. Ritchie, C.K., L.R. Andrews, K.G. Thomas, D.J. Tindall, and L.A. Fitzpatrick. 1997. The effects of growth factors associated with osteoblasts on prostate carcinoma proliferation and chemotaxis: Implications for the development of metastatic disease. Endocrinology 138:11451150.

4. Jiang, X., D.H. Rowitch, P. Soriano, A.P. McMahon, and H.M. Sucov. 2000. Fate of the mammalian cardiac neural crest. Development 127:1607-1616.

5. Morishita, R., H. Makino, M. Aoki, N. Hashiya, K. Yamasaki, J. Azuma, Y. Taniyama, Y. Sawa, et al. 2011. Phase $\mathrm{I} / \mathrm{Il}$ a clinical trial of therapeutic angiogenesis using hepatocyte growth factor gene transfer to treat critical limb ischemia. Arterioscler. Thromb. Vasc. Biol. 31:713-720.

6. Tateishi-Yuyama, E., H. Matsubara, and T. Murohara. 2002. Therapeutic angiogenesis for patients with limb ischemia by autologous transplantation of bone-marrow cells: a pilot study and a randomized controlled trial. ACC Curr. J. Rev. 11:86.

7. Kanegasaki, S., Y. Nomura, N. Nitta, S. Akiyama, T. Tamatani, Y. Goshoh, T. Yoshida, T. Sato, and Y. Kikuchi. 2003. A novel optical assay system for the quantitative measurement of chemotaxis. J. Immunol. Methods 282:1-11.

8. Zhao, M., B. Song, J. Pu, T. Wada, B. Reid, G. Tai, F. Wang, A. Guo, et al. 2006. Electrical signals control wound healing through phosphatidylinositol-3-OH kinase-gamma and PTEN. Nature 442:457-460.

9. Breckenridge, M.T., T.T. Egelhoff, and H. Baskaran. 2010. A microfluidic imaging chamber for the direct observation of chemotactic transmigration. Biomed. Microdevices 12:543-553.

10. Bambousková, M., Z. Hájková, P. Dráber, and P. Dráber. 2014. Basophils and Mast Cells. Methods Mol. Biol. 1192:161-176.

11. Decaestecker, C., O. Debeir, P. Van Ham, and R. Kiss. 2007. Can anti-migratory drugs be screened in vitro? A review of 2D and 3D assays for the quantitative analysis of cell migration. Med. Res. Rev. 27:149-176.

12. Feder-Mengus, C., S. Ghosh, A. Reschner, I. Martin, and G.C. Spagnoli. 2008. New dimensions in tumor immunology: what does 3D culture reveal? Trends Mol. Med. 14:333-340.

13. Even-Ram, S. and K.M. Yamada. 2005. Cell migration in 3D matrix. Curr. Opin. Cell Biol. 17:524-532.
14. Sabeh, F., I. Ota, K. Holmbeck, H. BirkedalHansen, P. Soloway, M. Balbin, C. Lopez-Otin, S. Shapiro, et al. 2004. Tumor cell traffic through the extracellular matrix is controlled by the membraneanchored collagenase MT1-MMP. J. Cell Biol. 167:769-781.

15. Ehrbar, M., A. Sala, P. Lienemann, A. Ranga, K. Mosiewicz, A/Bittermann, S.C. Rizzi, F.E. Weber, and M.P. Lutolf. 2011. Elucidating the role of matrix stiffness in 3D cell migration and remodeling. Biophys. J. 100:284-293.

16. Cukierman, E., R. Pankov, D.R. Stevens, and K.M. Yamada. 2001. Taking cell-matrix adhesions to the third dimension. Science 294:1708-1712.

17. Gadea, G., M. De Toledo, C. Anguille, and P. Roux 2007. Loss of p53 promotes RhoA-ROCK-dependent cell migration and invasion in 3D matrices. J. Cell Biol. 178:23-30.

18. Justice, B.Z., N.A. Badr, and R.A. Felder. 2009. $3 D$ cell culture opens new dimensions in cell-based assays. Drug Discov. Today 14:102-107.

19. Vernon, R.B. and M.D. Gooden. 2002. New technologies in vitro for analysis of cell movement on or within collagen gels. Matrix Biol. 21:661-669.

20. Vedula, S.R.K., G. Peyret, I. Cheddadi, T. Chen, A. Brugués, H. Hirata, H. Lopez-Menendez, Y. Toyama, et al. 2015. Mechanics of epithelial closure over non-adherent environments. Nat. Commun. 6:6111.

21. Shin, Y., S. Han, J.S. Jeon, K. Yamamoto, L.K. Zervantonakis, R. Sudo, R.D. Kamm, and S. Chuung. 2012. Microfluidic assay for simultaneous culture of multiple cell types on surfaces or within hydrogels. Nat. Protoc. 7:1247-1259.

22. Wiggins, H. and J. Rappoport. 2010. An agarose spot assay for chemotactic invasion. Biotechniques 48:121-124.

23. Lutolf, M.P., and J.A. Hubbell. 2003. Synthesis and physicochemical characterization of end-linked poly(ethylene glycol)-co-peptide hydrogels formed by Michael-type addition. Biomacromolecules 4:713-722.

24. Bracher, M., D. Bezuidenhout, M.P. Lutolf, T. Franz, M. Sun, P. Zilla, and N.H. Davies. 2013. Cell specific ingrowth hydrogels. Biomaterials 34:67976803.

25. Malpasso, G., M.C. Ciuffreda, F. Copes, K. Goetsch, D. Bezuidenhout, M. Mura, F. Pisano, N.H. Davies, and M. Gnecchi. 2015. Novel degradable hydrogel containing heparin improves the therapeutic effect of mesenchymal stromal cells for the treatment of myocardial infarction. J. Am. Coll. Cardiol. 65:A850.

26. Johnson, N.R., M. Kruger, K.P. Goetsch, P. Zilla D. Bezuidenhout, Y. Wang, and N.H. Davies. 2015. Coacervate Delivery of Growth Factors Combined with a Degradable Hydrogel Preserves Heart Function after Myocardial Infarction. ACS Biomater. Sci. Eng. 1:753-759.

27. Postlethwaite, A.E., J. Keski-oja, H.L. Moses, and A.H. Kang. 1987. Stimulation of the chemotactic migration of human fibroblasts by transforming growth factor beta. J. Exp. Med. 165:251-256.

Received 27 August 2015; accepted 09 May 2016.

Address correspondence to Neil Hamer Davies, Cardiovascular Research Unit, Chris Barnard Division of Cardiothoracic Surgery, University of Cape Town, Department of Health Sciences, Cape Town, South Africa. E-mail: neil.davies@uct.ac.za

To purchase reprints of this article, contact: biotechniques@fosterprinting.com 